\title{
Shedding Light on Thermally Induced Optocapacitance at the Organic Biointerface
}

\author{
Gaia Bondelli, Samim Sardar, Greta Chiaravalli, Vito Vurro, Giuseppe Maria Paternò,* \\ Guglielmo Lanzani,* and Cosimo D’Andrea*
}

Cite This: J. Phys. Chem. B 2021, 125, 10748-10758

Read Online

ABSTRACT: Photothermal perturbation of the cell membrane is typically achieved using transducers that convert light into thermal energy, eventually heating the cell membrane. In turn, this leads to the modulation of the membrane electrical capacitance that is assigned to a geometrical modification of the membrane structure. However, the nature of such a change is not understood. In this work, we employ an all-optical spectroscopic approach, based on the use of fluorescent probes, to monitor the membrane polarity, viscosity, and order directly in living cells under thermal excitation transduced by a photoexcited polymer film. We report two major results. First, we show that rising temperature does not just change the geometry of the membrane but indeed it affects the membrane dielectric characteristics by water penetration. Second, we find an

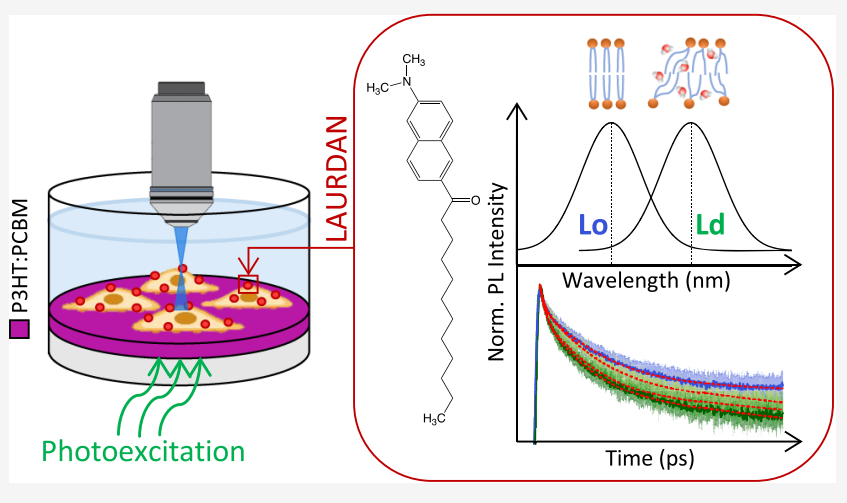
additional effect, which is peculiar for the photoexcited semiconducting polymer film, that contributes to the system perturbation and that we tentatively assigned to the photoinduced polarization of the polymer interface.

\section{INTRODUCTION}

The possibility to control selectively the electrical activity of living cells with low invasiveness has opened up new therapeutic paths in neurodegenerative medicine. ${ }^{1}$ Optical technologies are particularly suited for this purpose, as light enables precise and localized perturbation of cell activity in a remote and spatiotemporal precise manner. The cell membrane is the natural target for this approach. ${ }^{2-5}$ In particular, membrane potential modulation occurs via the photoinduced modification of the cell membrane electrical properties, such as resistance, capacitance, and resting potential, $^{2}$ either through direct photostimulation ${ }^{6}$ or using selected transducers that are able to convert light into an electrical, mechanical, chemical, or thermal stimulus. ${ }^{7-11}$ In the last decade, organic semiconductors have emerged as powerful photoactuators for the development of functional interfaces with living cells and organisms. These systems, which have benefited from a long period of development and refinement for applications in organic photovoltaics, ${ }^{12}$ exhibit a relatively high absorption coefficient $\left(\alpha \sim 10^{5} \mathrm{~cm}^{-1}\right)$ in the visible spectral range, which is suitable for light biostimulation. In addition to this functional quality, another important advantage is represented by the fact that organic semiconductors are essentially biomimetic compounds, being mostly composed of hydrogen and carbon atoms as it happens for biomolecules. This allows establishing a virtually seamless interface with biomolecules where both ionic and electronic processes can play a role. ${ }^{13}$ Taken together, these features make them ideal candidates for noninvasive optostimulation, for both in vitro and in vivo applications. ${ }^{9,10,14-22}$ Driven by these strong motivations, many efforts have been dedicated to shed light on the biophysics underpinning photostimulation with organic semiconductors. ${ }^{2,16,23-25}$

Here, we focus our attention onto a regime of abiotic/biotic interaction that takes place for relatively high light intensity of excitation $\left(>1 \mathrm{~mW} / \mathrm{mm}^{2}\right)$ and long interaction time (seconds). Under such conditions, the local rise in temperature is the main phenomenon driving membrane potential dynamics. Direct photothermal stimulation was first demonstrated using infrared light that heats up water, ${ }^{6}$ an effect that leads to a rapid local temperature rise and a consequent increase of membrane capacitance, which is associated with a transient depolarization. However, this method can be rather spatially imprecise because of the nonlocalized water absorption process and, in addition, can lead to long-term photodegradation

Received: July 7, 2021

Revised: August 19, 2021

Published: September 15, 2021 
effects. For these reasons, various light-to-heat transducers able to confine spatially the thermal effect have been recently proposed. For instance, gold quantum dots have been employed for these purposes, owing to their large plasmonic oscillator strength and their biocompatibility. ${ }^{26}$ In organic semiconductors, which represent the subject of our investigations, the heating process originates from the nonradiative recombination of the photogenerated states. If we consider a planar abiotic/biotic interface, in which living cells are cultured on top of a conjugated polymer film, the local temperature at the interface can be increased of several degrees by stimulation in the absorption region of the polymer and with light densities $>1 \mathrm{~mW} / \mathrm{mm}^{2}$. Within this context, Martino et al. observed a photoinduced increase in membrane capacitance after excitation of poly(3-hexylthiophene) (P3HT) alone and in blend with the electron-acceptor [6,6]-phenyl-C61-butyric acid methyl ester (PCBM), which was linked to an increase of the local temperature. ${ }^{16}$ In addition, the authors observed that ion channel conductance was also affected, leading to a two-phase response consisting of a depolarization followed by a hyperpolarization. $^{16^{\circ}}$

Regardless of the origin of the temperature increase, the corresponding increment in cell membrane capacitance emerges as a universal behavior, with a rate of $\sim 0.3 \% /{ }^{\circ} \mathrm{C}$. According to the simple plane capacitor model, a geometrical interpretation of the phenomenon based on thermal expansion would lead to an opposite outcome, namely, a capacitance decrease. Shoham et al. ${ }^{27}$ reconceived the model used by Shapiro ${ }^{6}$ introducing the ad-hoc conjecture of a thermally induced phase transition ${ }^{28}$ leading to the increase in capacitance. Indeed, under physiological conditions cell membranes are close to the phase transition temperature, and a slight temperature increase might lead to a structural change from gel to liquid phase of the cellular membrane bilayer. ${ }^{29}$ For example, the tilting of the lipid tails would reduce the membrane thickness and increase the membrane area, supporting the geometrical interpretation. ${ }^{30}$ The change in morphology may however be of different nature, that is, associated with the modification of the membrane composition, while the variation of membrane capacitance can be related to a change of the dielectric constant. Hence, the exact stimulation mechanism is still a matter of debate.

In this work, we employ an all-optical spectroscopic approach to shed light on these aspects. By probing the membrane polarity, viscosity, and order in living cells via wellestablished fluorescent probes, we provide solid evidence for the temperature-induced structural modification occurring in the membrane. Additionally, our results suggest that water permeates more favorably the disordered phase, and thus the resulting change in the dielectric response of the plasma membrane might well be the ruling phenomenon explaining the temperature-induced enhancement in capacitance. Furthermore, we find experimental evidence that optostimulation mediated by a photovoltaic organic film brings about an additional phenomenon reinforcing the perturbation effect. We tentatively assigned this to the surface charging at the biointerface. Our results suggest that optostimulation mediated by organic semiconductors is not simply due to thermal effects, but it is also related to the capability of the photogenerated charges to polarize the plasma membrane.

\section{METHODS}

\section{SUBSTRATE PREPARATION}

Regioregular P3HT (99.995\% purity, $M_{\mathrm{n}} \approx 54,000-75,000$ ) was purchased from Sigma-Aldrich; PCBM [C70] (99.5\% purity) was purchased from Nano-C; they were both used without any further purification. The substrates for cell cultures consisted of round glass coverslips (diameter $18 \mathrm{~mm}, \mathrm{VWR}$ ) and carefully rinsed in a successive ultrasonic bath of nanopure water, acetone, and isopropanol. A P3HT:PCBM (1:1 wt) solution was prepared in chlorobenzene at a final concentration of $10 \mathrm{mg} / \mathrm{mL}$. It was spin-coated on the cleaned substrates with a two-step procedure: (i) $3 \mathrm{~s}$ at $800 \mathrm{rpm}$ and (ii) $60 \mathrm{~s}$ at $1600 \mathrm{rpm}$, resulting in a final film thickness of around $100 \mathrm{~nm}$. For specific control measurements, the coloring pigment CI 15850 (D\&C Red No.7) was dissolved in $n$-butyl acetate, and its deposition process was carefully controlled to obtain films with comparable optical density at the selected excitation wavelength $(\lambda=561 \mathrm{~nm}$, see Figure S1). All films were thermally treated in an oven at $120{ }^{\circ} \mathrm{C}$ for 2 $\mathrm{h}$ for annealing and sterilization. To promote adhesion, samples for cell cultures were coated with fibronectin (from bovine plasma, Sigma-Aldrich) at a concentration of $2 \mu \mathrm{g} / \mathrm{mL}$ in phosphate buffered saline (PBS) for at least $30 \mathrm{~min}$ at $37^{\circ} \mathrm{C}$ and then rinsed with PBS.

Cell Culture Maintenance. Both confocal imaging and time-resolved photoluminescence (TRPL) in vitro measurements were performed using the immortalized cell line HEK293 (human embryonic kidney), purchased from ATCC. HEK-293 cells were cultured in T-75 cell culture flasks containing Dulbecco's modified Eagle's medium-high glucose (DMEM-HG), supplemented with $10 \%$ heat-inactivated fetal bovine serum (Gibco, UK), 2\% penicillin/streptomycin, and $1 \%$ L-glutamine. Phenol red-free medium was preferred, to reduce background fluorescence in subsequent experiments. Culture flasks were maintained in a humidified incubator at 37 ${ }^{\circ} \mathrm{C}$ with $5 \% \mathrm{CO}_{2}$. When at confluence, cells were enzymatically dispersed with a $1 \mathrm{x}$ trypsin-EDTA solution, plated on the substrates, and left to grow.

TRPL Setup. In the setup used for TRPL measurements, the light source was provided by a Ti:Sapphire oscillator (Chameleon Ultra II, Coherent) producing pulses of $140 \mathrm{fs}$ with a repetition rate of $80 \mathrm{MHz}$. Second harmonic generation was obtained using a barium borate crystal; two absorbing BG40 filters removed residuals of the fundamental. Spatial resolution was achieved through a microscope incorporated in the setup. The excitation beam $(370 \mathrm{~nm})$ was reflected off a suitably chosen dichroic mirror (LP435) before being coupled into the objective and focused onto the sample, obtaining an excitation spot diameter of $4-5 \mu \mathrm{m}$ and an average power of $25 \mu \mathrm{W}$. To achieve high excitation efficiency, a 63x water immersion objective (Leica HCX APO L 63X) with a high numerical aperture (NA 0.9) was required. Sample emission was collected by the same objective and transmitted through the dichroic mirror, and furthermore, an LP395 absorbing filter was used to remove the residual pump scatter. The microscope field of view was selected by a flip mirror and a CMOS camera (ORCA-Flash 2.8, Hamamatsu), allowing accurate positioning of the sample relative to the excitation beam via a sample XYZ differential micrometer translation stage. The emission signal was focused on the entrance slit of a spectrograph (Acton SP2300i, Princeton Instrument). The dispersed image was 
then focused on the entrance slit of a streak camera (Hamamatsu C5680), equipped with the Synchroscan sweep module. For Laurdan measurements, the maximum voltage applied to the sweep electrodes was such as the temporal window acquired had a time width of $2 \mathrm{~ns}$, with an instrument response function of $\sim 20$ ps. A CCD (Hamamatsu ORCA-R2 C10600) recorded the streak image. For the simultaneous excitation of the P3HT:PCBM film, a $561 \mathrm{~nm}$ continuous wave (CW) DPSS laser (SLIM-561-150, Oxxius) was employed, whose beam was collimated to a final diameter of $1 \mathrm{~mm}$, striking the sample from the bottom (see Figure 3c). Its power density was adjusted by placing an optical attenuator in the excitation path. To avoid photoluminescence (PL) contribution from the CW laser and the polymeric film, notch 561 and FES550 filters were used in the collection path.

Confocal Imaging and Spectroscopic Measurements with Laurdan. Laurdan (6-lauryl-2-dimethylamino-napthalene) was purchased from Sigma-Aldrich, UK. For confocal imaging and TRPL measurements, a $5 \mathrm{mM}$ stock solution of Laurdan in dimethyl sulfoxide (DMSO, Sigma-Aldrich, UK) was prepared. Laurdan staining of live HEK-293 cells was performed removing the medium from the cell culture dish, prepared as described in the cell culture section above, and replacing it with $1 \mathrm{~mL}$ of fresh, serum-free DMEM, as the presence of serum increases background fluorescence and sequesters the dye during incubation. Laurdan was then added at a final concentration of $10 \mu \mathrm{M}$, known to efficiently stain cells without affecting their viability. ${ }^{31}$ Cells were incubated at $37{ }^{\circ} \mathrm{C}$ under a humidified $5 \% \mathrm{CO}_{2}$ atmosphere for $30 \mathrm{~min}$ in the dark. For confocal microscopy measurements, Laurdan was incubated for much longer, to have an overview of the full cellular membranous environment, including the intracellular membranes. After incubation, cells were washed with warmed PBS to remove cell-unbound molecules and ready for measurements.

For confocal imaging of Laurdan-stained cells, samples were mounted on an inverted confocal laser-scanning microscope (Nikon Eclipse Ti2, Nikon Instruments), and acquisitions were performed using an Olympus $60 \times$ oil objective. Cells were excited with a $403 \mathrm{~nm}$ diode laser, and the fluorescence was collected in two detection channels: $425-475 \mathrm{~nm}$ and 500$550 \mathrm{~nm}$. Confocal images were analyzed with Fiji (ImageJ), and the generalized polarization (GP) fluidity map was realized through MATLAB.

For TRPL measurements, samples were placed on the stage of the TRPL microscope, and Laurdan PL was obtained under excitation at $370 \mathrm{~nm}$. Each measurement lasted no more than 2 $\mathrm{min}$, to reduce cellular stress during excitation. Because of high intrinsic cell membrane heterogeneity, the recorded spectra and dynamics slightly differ from cell to cell and from different spots of the same cell. For this reason, the cell area of interest was fixed while performing consecutive measurements at increasing temperatures, to avoid influencing the Laurdan response by changing the cell spot under investigation. The shape and general aspect of each sampled cell were checked after each measurement through the wide-field CMOS camera, to ensure that the cell did not undergo any change in shape or position. For each sample, three-four measurements were performed, and the samples were changed after about $40 \mathrm{~min}$ from the end of Laurdan incubation, to avoid excessive internalization. TRPL measurements at different temperatures were carried out placing the sample chamber on top of a commercial Peltier plate, whose voltage was adjusted via a source meter (Agilent, B2912A). Emission spectra were fitted with two Gaussians accounting for the Lo and Ld phases, whose center $\left(x_{\mathrm{c}}\right)$ was fixed to 460 and $510 \mathrm{~nm}$, respectively. For the GP analysis, formula 5 was adjusted to the following one:

$$
\mathrm{GP}=\frac{I_{(460)}-I_{(510)}}{I_{(460)}+I_{(510)}}
$$

where $I$ corresponds to the area underneath the Gaussian curve in consideration. The statistic was made on about six different cells per each sample condition.

Anisotropy Fluorescence Measurements with TMADPH. 1-(4-trimethylamino)phenyl)-6-phenylhexa-1,3,5-triene (TMA-DPH) was purchased from Invitrogen. Introduced by Prendergast et al. ${ }^{32}$ in 1981 as a model molecular rotor for phospholipidic membranes, TMA-DPH interacts with living cells by instantaneous partition between the plasma membrane and the external medium. With fluorescence anisotropy, membrane fluidity is interpreted in terms of hindrance to rotational motion of the probe, because it is embedded in the constraining phospholipidic membrane array. ${ }^{33}$ To measure the decay of fluorescence anisotropy with TMA-DPH, polarizers were placed in the excitation and emission path. Decays of vertically, $I(t)_{\mathrm{vv}}$, and horizontally, $I(t)_{\mathrm{vh}}$, polarized fluorescence intensities with respect to the excitation beam were measured. Time-dependent anisotropy was calculated as:

$$
r(t)=\frac{I(t)_{\mathrm{vv}}-I(t)_{\mathrm{vh}}}{I(t)_{\mathrm{vv}}+2 I(t)_{\mathrm{vh}}}
$$

where $I(t)_{\mathrm{vv}}$ and $I(t)_{\mathrm{vh}}$ represent the components of the light intensity emitted, respectively, parallel and perpendicular to the direction of the vertically polarized excitation light. For these fluidity measurements, HEK-293 cells were incubated with TMA-DPH for a short time (10 s) at room temperature, to label the plasma membrane. The final concentration of the probe in PBS was $2 \mu \mathrm{M}$, starting from a $5 \mathrm{mM}$ stock solution in dimethylformamide.

Photovoltage (PV) Measurements. Electrochemical measurements have been conducted using a potentiostat and an electrochemical cell in a three-electrode setup. The potentiostat used was a Metrohm PGSTAT302N. The reference electrode was saturated $\mathrm{Ag} / \mathrm{AgCl}$ (Metrohm 60733100), and the counter electrode was a Pt wire (Metrohm 60301100). Samples were connected as the working electrode using crocodile clips in direct contact with ITO over a portion of the sample not covered by the P3HT:PCBM film. The used electrolytic solution was PBS. The illumination was supplied by a green light-emitting diode (LED) (Thorlabs M530L3-C5, $530 \mathrm{~nm}$ ), driven by a DC2200 LED driver. The LED was placed at a distance of $25 \mathrm{~cm}$ from the sample and operated at maximum power. The intensity of light hitting the sample was $500 \mathrm{~W} / \mathrm{m}^{2}$. PV recordings were acquired operating the potentiostat in high-speed mode using a sample rate of $10^{4}$ $\mathrm{s}^{-1}$. PV curves were all zeroed to the open-circuit potential value.

Zeta Surface Potential Measurements. Zeta surface potential measurements have been conducted with a Zetasizer Nano with the surface zeta potential cell with a height adjustable sample holder. The cell was submerged in a solution containing silica nanoparticles, used as tracers. Measurements were performed at $125,250,375,500$, and $625 \mu \mathrm{m}$ from the P3HT:PCBM sample surface. A final measurement at $1000 \mu \mathrm{m}$ 
was also performed to estimate the tracer potential only because of the electrophoretic effect. The surface zeta potential was then computed as:

$$
\zeta_{\{\text {wall }\}}=- \text { intercept }+\zeta_{\{\text {tracer }\}}
$$

where $\zeta_{\{\text {wall }\}}$ is the surface potential at the interface between P3HT:PCBM and electrolyte, intercept is the linear regression of the distance-dependent data up to the zero displacement point, and $\zeta_{\text {\{tracer }}$ is the potential of the silica nanoparticles without any contribution from the potential of the surface.

Drift-Diffusion Simulations. The theoretical work was based on the use of a time-dependent version of the driftdiffusion model to describe the transport mechanisms of P3HT:PCBM in 1D. The mathematical model consists of two continuity equations accounting for the drift and diffusion of photogenerated holes and electrons and a Poisson equation for the electric field and potential. The computational algorithm was coded in-house and has been implemented using the MATLAB scientific environment. The generation of charge carriers is described with the use of the Lambert Beer law, while the recombination process is described with the Langevin model. At $x=0$, the evaluation of the PV is performed, defined as $\mathrm{PV}=\psi(x=0)$, where $\psi$ is the electric potential. The boundary condition at the interface between P3HT:PCBM and the electrolyte is described using MarcusGerischer theory:

$$
J_{\text {cat }}=\frac{q k_{\mathrm{t}} c^{\mathrm{ox}} N_{\mathrm{A}} \sigma}{\left(\pi k_{\mathrm{B}} T \lambda\right)^{1 / 2}} n \exp \left(-\frac{\left(E_{\mathrm{L}}-E_{\mathrm{F}}^{\mathrm{OX}}-\lambda\right)^{2}}{4 \lambda k_{\mathrm{B}} T}\right)
$$

where $q$ is the elementary charge $[\mathrm{C}], k_{\mathrm{t}}$ is the tunneling constant $\left[\mathrm{m}^{4} \mathrm{~s}^{-1}\right], c^{\text {ox }}$ is the concentration of molecular oxygen at the interface $\left[\mathrm{mol} \mathrm{m}{ }^{-3}\right],{ }^{34} N_{\mathrm{A}}$ is the Avogadro constant $\left[\mathrm{mol}^{-1}\right], \sigma$ is the disorder parameter of $\mathrm{P} 3 \mathrm{HT}[\mathrm{eV}], k_{\mathrm{B}}$ is the Boltzmann constant $\left[\mathrm{J} \mathrm{K}^{-1}\right], T$ is the room temperature $[\mathrm{K}]$, and $\lambda$ is the width of the Gaussian distribution of molecular oxygen states $[\mathrm{eV}] . E_{\mathrm{L}}$ is the energy of the LUMO and $E_{\mathrm{F}}^{\mathrm{OX}}$ is the energy corresponding to the potential of the oxygen reduction reaction. $n$ is the electron number density at $x=L$ $\left[\mathrm{m}^{-3}\right]$. The surface charge accumulated at the interface provides surface recombination centers for the holes of P3HT:PCBM. The time constant of this effect and $k_{\mathrm{t}}$ have been estimated from the fitting with the experimental data. See the Supporting Information for more details.

\section{RESULTS}

Optical Probing of Membrane Fluidity and Order. Biological membranes are complex systems composed of a wide variety of lipids, sterols, and proteins, ${ }^{35,36}$ that can exist in a variety of phases, from liquid-ordered (Lo) to liquiddisordered (Ld). Different phases coexist in the membrane, and interchanging between them occurs at physiologically relevant temperatures. ${ }^{37-40}$ A broad term commonly adopted to describe the physical state of biological membranes is fluidity, referring to the overall Lo/Ld membrane phase ratio. The maintenance of physiological cell membrane fluidity is a prerequisite for proper membrane function, as it is associated with cell viability and normal cell growth and division. ${ }^{41}$ In addition, this parameter influences the dynamics of membrane proteins, ${ }^{42}$ which in turn affects the likelihood of proteinprotein interactions and hence the efficiency of signaling pathways. Fluidity can be associated with the degree of lipid packing, the thickness of the lipid bilayer, and the rotational freedom of lipids. ${ }^{31}$ The degree of lipid packing can be assessed from the polarity of the bilayer. A more compact lipid arrangement excludes polar water molecules from the otherwise nonpolar bilayer, resulting in a lowering of local environmental polarity, which can ultimately be evaluated by employing polarity-sensitive fluorescent probes. In this regard, one of the most popular and established fluorescence probes is Laurdan (Figure 1a). ${ }^{31,43}$ Laurdan is an amphiphilic polarity-
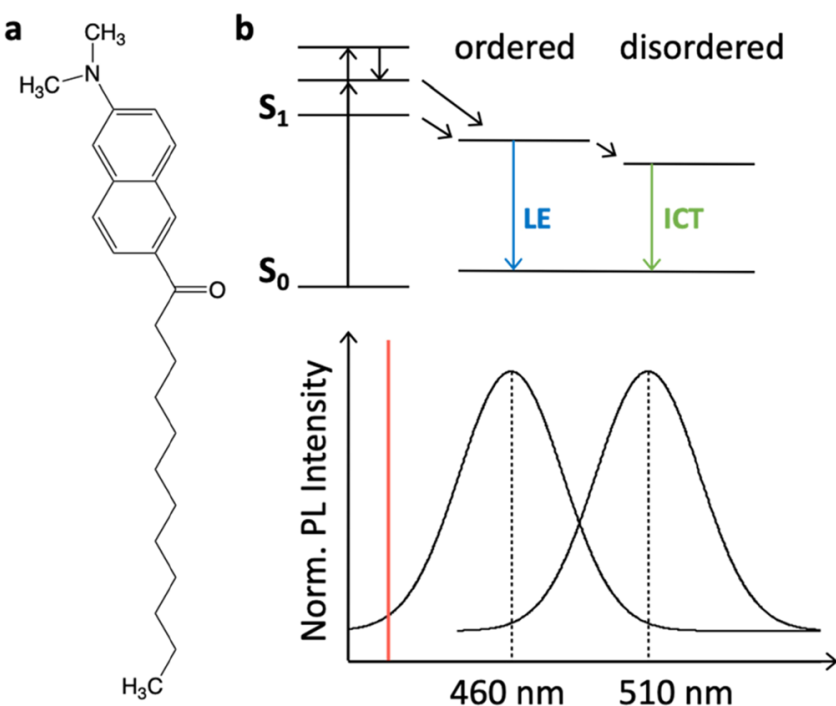

Figure 1. Laurdan as a polarity-sensitive probe. (a) Laurdan chemical structure. (b) Laurdan Jablonski diagram, illustrating absorption (exc. $370 \mathrm{~nm}$, red line), solvent relaxation to, and emission from two different excited states: LE state, with emission near $460 \mathrm{~nm}$, and ICT state in more polar environments, with emission at longer $\lambda$. A schematic representation of the corresponding emission maxima of Laurdan in ordered and disordered phases is shown.

sensitive dye, intrinsically nonfluorescent in water. When intercalates into the membrane bilayer, the molecule is however fluorescent with at least two excited states (Figure 1b): an intrinsic locally excited (LE) state and an internal charge transfer (ICT) state characterized by a large permanent dipole moment. The latter is stabilized by the reorientation of the surrounding water molecules with the Laurdan dipole moment, ${ }^{44}$ so that the frequency of the emitted photons is decreased. ${ }^{44}$ Thus, the emission spectrum of the dye undergoes a shift to longer wavelengths in more polar environments. ${ }^{45-49}$ This property, commonly known as solvent relaxation, ${ }^{50}$ makes such solvatochromic dye perfect for probing lipid phases, as the more loosely packed Ld phase presents much higher hydration than the tightly packed Lo phase, resulting in a significant fluorescence spectrum shift. Moreover, Laurdan partitions equally between Lo and Ld, giving comparable quantum yields in both phases. ${ }^{51,52}$ The emission lineshape allows a quantitative assessment of the membrane order that is traditionally expressed by the $\mathrm{GP}^{31,53}$ parameter, which is defined as:

$$
\mathrm{GP}=\frac{I_{\mathrm{B}}-I_{\mathrm{G}}}{I_{\mathrm{B}}+I_{\mathrm{G}}}
$$

where $I_{\mathrm{B}}$ and $I_{\mathrm{G}}$ correspond to the intensities at the blue and green edges of the emission spectrum, respectively, for a given excitation wavelength. ${ }^{54}$ Because the GP value is a ratio of two intensities, measurements do not depend on local probe 

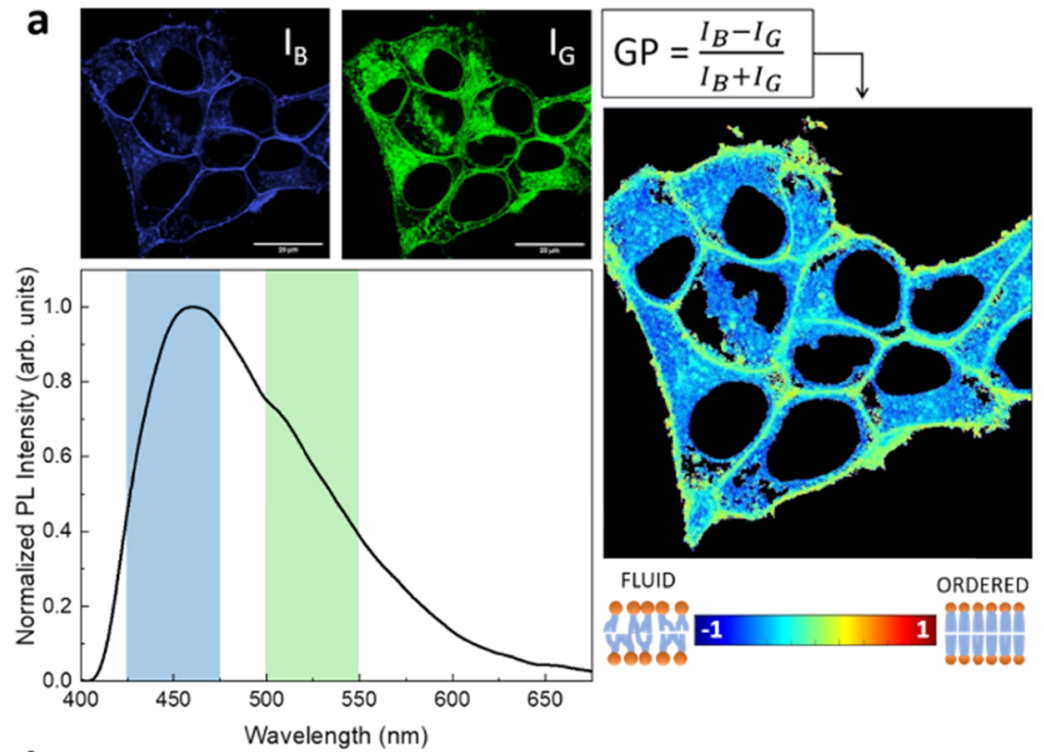

b

C
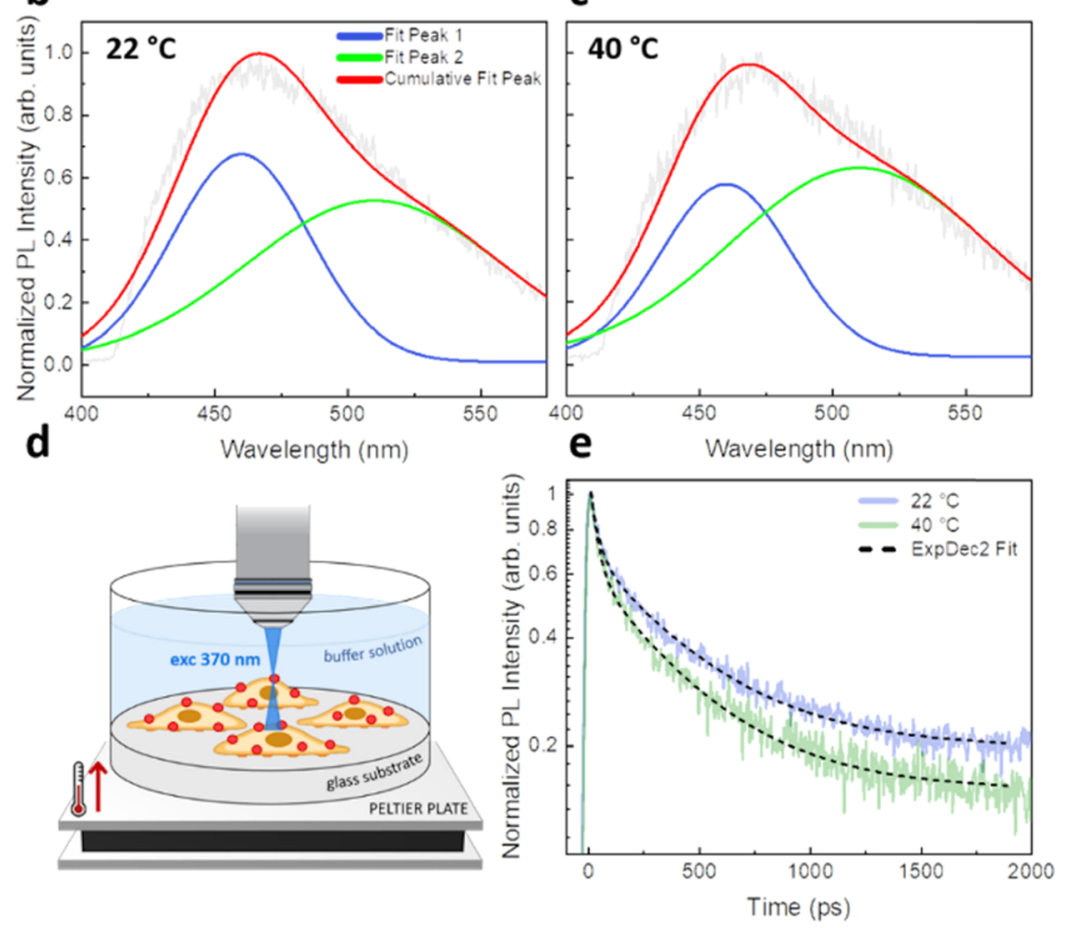

Figure 2. Laurdan probe monitors membrane fluidity in cells through a shift in the emission spectrum. (a) Laurdan fluorescence images of HEK293 cells recorded simultaneously in the blue channel $\left(425-475 \mathrm{~nm}, I_{\mathrm{B}}\right)$ and green channel $\left(500-550 \mathrm{~nm}, I_{\mathrm{G}}\right)$ and relative Laurdan emission spectrum. On the right, fluidity map obtained applying the GP formula for each pixel, pseudocolored as indicated by the scale with GP ranging from -1 to 1 . The scale bar is $20 \mu \mathrm{m}$. (b-e) TRPL measurements on HEK-293 cells grown on glass substrates. Deconvolution of the Laurdan emission spectra in HEK-293 cells recorded at $22{ }^{\circ} \mathrm{C}$ (b) and $40{ }^{\circ} \mathrm{C}$ (c), upon excitation at $370 \mathrm{~nm}$, fitted with two Gaussian curves centered at 460 and 510 $\mathrm{nm}$, used in the GP evaluation. Obtained values go toward more negative values (disordered state). (d) Schematic representation of the sample used in this study, heated by a Peltier plate. (e) Laurdan PL kinetics, integrated in the range of $440-650 \mathrm{~nm}$, fitted to biexponential decay functions: dynamics become faster when temperature is increased.

concentrations or surface area. Importantly, the membranephase-dependent emission shifts have been reported to be independent of the nature of the glycerophospholipid polar head group. ${ }^{55}$

Even though steady-state GP measurements can provide useful estimates of the overall lipid environment, ${ }^{56}$ an analysis of the fluorescence lifetime of Laurdan can provide further information, as the local membrane environment affects the excited state dynamics. Laurdan excited states are in fact sensitive to collisional quenching by water molecules within the membrane..$^{57-59}$ Consequently, a decrease in Laurdan fluorescence lifetime can be assigned to an increase in water content of the membrane $e^{45,59}$ and, thus, to a more disordered phase.

We analyzed the fluorescence properties of Laurdan internalized in the membrane of live HEK-293 cells, grown on two different substrates: a calibration sample where the substrate is glass and the temperature increase is provided by 
the use of a Peltier plate; and a spin-casted film of P3HT:PCBM (1:1 wt) blend, which was photoexcited with a $\mathrm{CW}$ laser at $561 \mathrm{~nm}$. TRPL measurements with picosecond temporal resolution were carried out to record the entire time course of the Laurdan spectral shift immediately after excitation.

Confocal Imaging and TRPL Measurements on HEK293 Cells Plated on Glass Samples. As a preliminary analysis, we stained with Laurdan live HEK-293 cells plated on glass substrates and we observed them through a confocal microscope. Figure 2a depicts Laurdan fluorescence images of HEK-293 cells excited with a $403 \mathrm{~nm}$ laser and recorded them simultaneously in emission ranges of $425-475\left(I_{\mathrm{B}}\right)$ and 500$550 \mathrm{~nm}\left(I_{\mathrm{G}}\right)$. The corresponding Laurdan emission spectrum is also reported. In the HEK-293 cell membrane at room temperature $\left(22^{\circ} \mathrm{C}\right)$, Laurdan emission is composed of two spectral contributions centered at 460 and $510 \mathrm{~nm}$, accounting for the ordered and disordered lipid phases, respectively. As discussed in the Introduction, membrane fluidity is expressed in terms of ratio of emission intensities using the GP formula, which ranges from -1 (disordered environment) to +1 (ordered environment). Here, we calculated GP for each pixel to obtain a "fluidity map" (right side of Figure 2a), where blue colors indicate a low membrane order in correspondence of intracellular membranes, whereas yellowish colors indicate a higher membrane order, matching the plasma membrane, which indeed contains the majority of membrane cholesterol, appearing thus as a "more ordered" phase. These measurements validate the use of Laurdan and provide the background for further experiments.

Calibration of the Laurdan spectral changes at different temperatures was carried out in a more versatile TRPL setup, which allowed achieving intracellular spatial resolution and hence sample specific cell spots with a diameter of around 4-5 $\mu \mathrm{m}$ (cell size $\sim 10$ to $20 \mu \mathrm{m}$ ). The possibility to have a microscope in the upright configuration permitted to position a Peltier plate below the sample chamber (Figure 2d), whose temperature was controlled by a source meter. Upon increasing the sample temperature from 22 to $40{ }^{\circ} \mathrm{C}$, the $\mathrm{PL}$ spectra of Laurdan exhibit an increase of the spectral contribution peaking at $510 \mathrm{~nm}$, which is indicative of the transition to a more disordered state of the membrane. We deconvolved the experimental spectra into two Gaussian components, whose values are reported in Table S1. Figure $2 b, c$ shows the best fit recorded at 22 and $40{ }^{\circ} \mathrm{C}$ with excitation at $370 \mathrm{~nm}$. From these, we worked out the value of GP based on eq 3 to characterize the predominant phase state of the lipid domain. We found average GP starting values at $22{ }^{\circ} \mathrm{C}$ to be equal to 0.19 , which are consistent with previous studies on HEK-293 cells. ${ }^{60}$ The resulting $\Delta \mathrm{GP}=\mathrm{GP}\left(40{ }^{\circ} \mathrm{C}\right)-\mathrm{GP}\left(22^{\circ} \mathrm{C}\right)$ was equal to $-0.187 \pm 0.02(\Delta \mathrm{GP} / \mathrm{GP} \approx-1)$, indicating increased fluidity and membrane hydration upon increasing the temperature. Next, we measured the Laurdan PL lifetime upon changing temperature (Figure $2 \mathrm{e}$ ). The lifetime traces of the Laurdan-labeled plasma membrane could be well fitted to double exponential decay curves ${ }^{61,62}\left(\tau_{1}<\tau_{2}\right)$ plus an offset component $\left(y_{0}\right)$, accounting for the long component that falls outside our time-window ( $>2 \mathrm{~ns})$. We integrated the kinetics in the range of $440-650 \mathrm{~nm}$, avoiding a Raman peak associated with the aqueous buffer, centered at $430 \mathrm{~nm}$. Fitted values are reported in Table 1.

The fast component $\tau_{1}$ does not change, but it acquires a larger amplitude at higher temperature. Upon heating, $\tau_{2}$ drops
Table 1. Laurdan Decay Curve Average Parameters $(n=6)$ Obtained at 22 and $40{ }^{\circ} \mathrm{C}$ on HEK-293 Cells Plated on Glass Substrates ${ }^{a}$

\begin{tabular}{lll} 
& \multicolumn{1}{c}{$22{ }^{\circ} \mathrm{C}$} & \multicolumn{1}{c}{$40{ }^{\circ} \mathrm{C}$} \\
$y_{0}$ & $0.19 \pm 0.002$ & $0.15 \pm 0.002$ \\
$\tau_{1}$ & $35 \pm 5$ ps $(\sim 45 \%)$ & $35 \pm 5$ ps $(\sim 53 \%)$ \\
$\tau_{2}$ & $427 \pm 8$ ps $(\sim 55 \%)$ & $402 \pm 9$ ps $(\sim 47 \%)$
\end{tabular}

${ }^{a}$ Kinetics were fitted to biexponential decay functions, with an offset $\left(y_{0}\right)$ accounting for the long lifetime component that falls outside our time-window.

by almost $6 \%$ and shows a smaller relative weight, while the offset $y_{0}$ decreases $(-21 \%)$. We evaluated the adequacy of the biexponential decay fitting by inspection of the residual distribution and by the statistical parameter reduced chisquare $\left(\chi^{2}\right)$ (Figure S2). To summarize, our data indicate that cells at higher temperature $\left(40{ }^{\circ} \mathrm{C}\right)$ displayed a shorter Laurdan lifetime, in agreement with the GP spectral shift. This implies an increased quenching from water molecules at the membrane-water interface and thus an increased level of water hydration, consistent with the enhanced disorder (fluidity) highlighted by steady-state measurements. This result suggests that by enhancing temperature the polarity of the membrane is increased (i.e., its dielectric constant) because of the penetration of water in the membrane. This process contributes to the increase in cell membrane capacitance that is observed by heating.

TRPL Measurements on HEK-293 Cells Plated on P3HT:PCBM Samples. In the previous experiments, heating of the cell specimens was provided by the Peltier plate. Hereafter, we introduce a P3HT:PCBM thin layer spin-coated on top of the glass substrate as the photothermal agent, resulting in the same device structure employed for optical cell stimulation. $^{16}$ Upon resonant excitation $(561 \mathrm{~nm})$, the polymeric film acts as a light transducer that dissipates the great majority of the absorbed photons energy via nonradiative decay and ultimately by heat transfer to the surrounding environment. Note that the PL quantum yield of the bulk heterojunction film is very small, limiting possible interference with the detection of Laurdan emission. ${ }^{63}$ Using finite element simulations, we estimate that the temperature variation at the interface with the polymeric film induced by illumination of the sample $\left(A_{\text {light }}=0.78 \mathrm{~mm}^{2}, I_{\text {light }}=46 \mathrm{~mW} / \mathrm{mm}^{2}\right.$, see the Supplementary Information) is about $16{ }^{\circ} \mathrm{C}$ at the surface, bringing the system to a final temperature of around $37{ }^{\circ} \mathrm{C}$ (Figure S3). According to the simulation, temperature reaches a stationary value in about $20 \mathrm{~s}$, that is, at the beginning of the light exposure lasting $120 \mathrm{~s}$. To ensure that the underlying blend film is not contributing to Laurdan PL, we performed some control measurements with the P3HT:PCBM film and aqueous buffer solution only, without the presence of cells (Figure S4). In any case, we observed a PL signal from P3HT:PCBM only in the first $\sim 100$ ps, which we could easily discard in the subsequent analysis. We then analyzed Laurdan fluorescence properties in live HEK-293 cells grown on top of the P3HT:PCBM film, to assess directly any alterations in the membrane phase. Notice that we focus our attention onto the regime of long stimuli (seconds) and relatively high light intensity of excitation $\left(>1 \mathrm{~mW} / \mathrm{mm}^{2}\right)$, which may be different from the one adopted in electrophysiology measurements to test membrane capacitance changes. Under the conditions employed in this work, the local rise in temperature is the main 
a

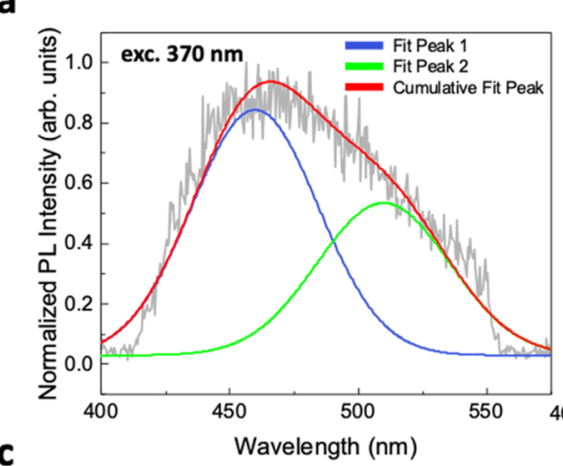

b

C
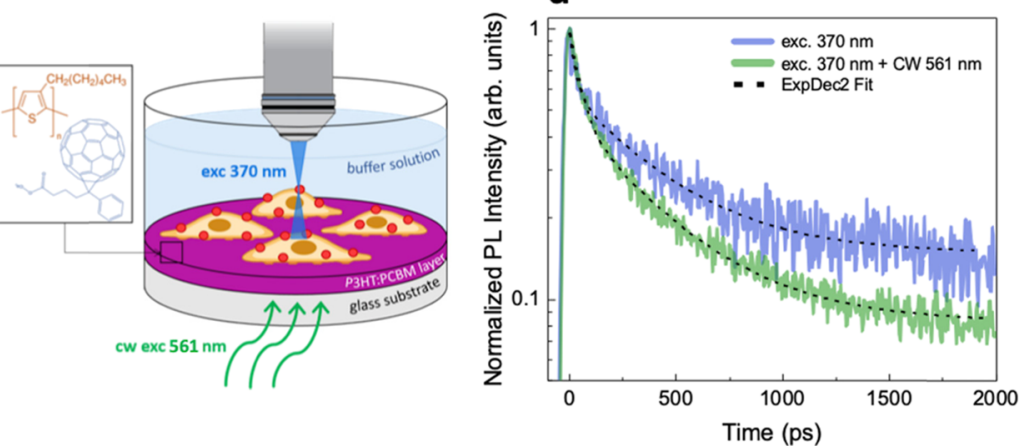

Figure 3. TRPL measurements on HEK-293 cells grown on P3HT:PCBM films. Deconvolution of the Laurdan emission spectra, recorded with excitation at $370 \mathrm{~nm}$ only (a) and at $370 \mathrm{~nm}+\mathrm{CW} 561 \mathrm{~nm}\left(46 \mathrm{~mW} / \mathrm{mm}^{2}\right)$ (b), fitted with two Gaussian curves centered at 460 and $510 \mathrm{~nm}$, used in the GP evaluation. Measurements were performed on the same cell spot. PL contribution from the polymeric film was avoided integrating only the range of 100-2000 ps. (c) Schematic representation of the sample used in this study. The laser beam illuminates the back of the sample, where the P3HT:PCBM film is located, while the PL excitation $370 \mathrm{~nm}$ beam impinges the sample from the opposite side, where the cells stained with Laurdan are plated. (d) Laurdan PL kinetics, integrated in the range of $440-550 \mathrm{~nm}$, fitted to double exponential decay curves: dynamics become faster upon illumination with a $561 \mathrm{~nm}$ CW laser.

phenomenon driving membrane potential dynamics. We first performed a control measurement heating the P3HT:PCBM film through the Peltier plate from 22 to $40{ }^{\circ} \mathrm{C}$, as previously done for the glass samples. The average values of the two Gaussian components measured are reported in Table S2. The worked out average $\Delta \mathrm{GP}$ was equal to $-0.152 \pm 0.03(\Delta \mathrm{GP} /$ $\mathrm{GP} \approx-0.97)$, very similar to the one obtained with glass samples. This allowed us to exclude possible influences of the substrate on the cell membrane ability to react to a direct temperature change. We then carried out consecutive measurements exciting first the sample with the $370 \mathrm{~nm}$ pulsed laser only and then with both $370 \mathrm{~nm}$ and CW $561 \mathrm{~nm}$ lasers at the same time (lasting $120 \mathrm{~s}$ ). As reported in Figure 3, we found that excitation of the P3HT:PCBM film leads to the enhancement of the green emission $(510 \mathrm{~nm})$. In this case, the average $\Delta \mathrm{GP}$ shifted toward more negative values $(\Delta \mathrm{GP}=$ $-0.349 \pm 0.04, \Delta \mathrm{GP} / \mathrm{GP} \approx-1.84$, Figure $3 \mathrm{a}, \mathrm{b}$ and Table S3), an effect which can be ascribed to a predominance of Ld lipid domains and increased membrane hydration. We note that the measured $\triangle G P$ s are almost double in magnitude when compared to those measured in the Peltier plate experiment, albeit the expected rise in temperature, for the applied power density (46 $\mathrm{mW} / \mathrm{mm}^{2}$ ) and time duration $(120 \mathrm{~s})$, is slightly less than the one obtained by Peltier heating $\left(16\right.$ vs $\left.18{ }^{\circ} \mathrm{C}\right)$. We then analyzed the variation in the PL dynamics (Figure $3 \mathrm{~d}$ ). Again, we found that the results follow the same trend as those collected on heated glass samples; however, the changes obtained switching on the $561 \mathrm{~nm} \mathrm{CW}$ laser are larger. We integrated the kinetics in the range of $440-550 \mathrm{~nm}$ and fitted to double exponential decay curves as before (see residual distribution in Figure S5). In the measured average values (Table 2 ), the time constant $\tau_{2}$ remarkably drops by $20 \%$, while $\tau_{1}$ remains constant and $\mathrm{y}_{0}$ decreases by almost $40 \%$.

Table 2. Laurdan Decay Curve Average Parameters $(n=6)$ Obtained Exciting HEK-293 Cells Plated on P3HT:PCBM Substrates $^{a}$

\begin{tabular}{lll} 
& \multicolumn{1}{c}{ exc. $370 \mathrm{~nm}$} & exc. $370 \mathrm{~nm}+$ CW $561 \mathrm{~nm}$ \\
$y_{0}$ & $0.15 \pm 0.003$ & $0.09 \pm 0.002$ \\
$\tau_{1}$ & $30 \pm 6 \mathrm{ps}(\sim 46 \%)$ & $30 \pm 6 \mathrm{ps}(\sim 51 \%)$ \\
$\tau_{2}$ & $388 \pm 18$ ps $(\sim 54 \%)$ & $310 \pm 6$ ps $(\sim 49 \%)$
\end{tabular}

${ }^{a}$ Kinetics were fitted to biexponential decay functions, with an offset $\left(y_{0}\right)$ accounting for the long lifetime component that falls outside our time-window.

We checked that the use of the $\mathrm{CW}$ laser without the underlying P3HT:PCBM layer does not lead to any detectable modification of the Laurdan emission (see Figure S6). Figure S7 shows that the photoinduced changes induced by P3HT:PCBM survive after switching off the laser light. Finally, we measured $\Delta \mathrm{GP}$ using several power densities of the $561 \mathrm{~nm}$ light $\left(29,17,8.6\right.$, and $\left.4.5 \mathrm{~mW} / \mathrm{mm}^{2}\right)$. The results show that $\Delta \mathrm{GP}$ is always negative and roughly linearly dependent on laser intensity (Figure 4a). In addition, Laurdan emission decay is consistently faster upon increasing intensity (Figure $4 \mathrm{~b}$ ). The results of the double exponential fitting of the decay curves are reported in Table S4. Note that the presence of the $370 \mathrm{~nm}$ light might lead to some heating mediated by the absorbing polymer film (a rough estimate of the upper limit is 

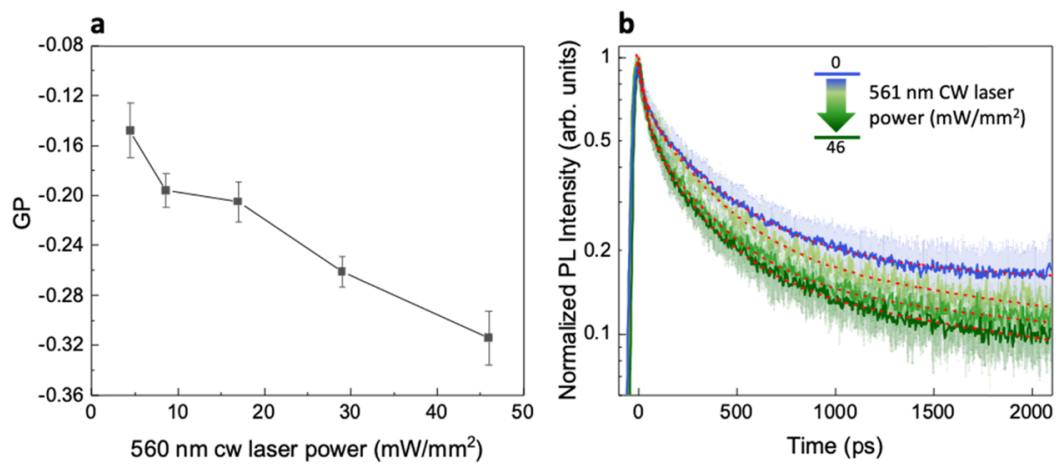

Figure 4. Laurdan PL as a function of $561 \mathrm{~nm} \mathrm{CW}$ laser power. (a) Average $(n=6) \Delta$ GP for TRPL measurement with $370 \mathrm{~nm}$ excitation only and TRPL measurement with $370 \mathrm{~nm}+\mathrm{CW} 561 \mathrm{~nm}$ excitation; error bars represent the standard deviation. (b) Laurdan TRPL decays at increasing $561 \mathrm{~nm} \mathrm{CW}$ laser power: dynamics become faster, going from $0 \mathrm{~mW} / \mathrm{mm}^{2}$ (exc. $370 \mathrm{~nm}$ only) to $46 \mathrm{~mW} / \mathrm{mm}^{2}$ and were fitted to double exponential decay curves.

$\left.1^{\circ}\right)$. Yet what we measure is a change on top of this contribution, if any. In addition, if the $370 \mathrm{~nm}$ light were effective, we would detect a change in the GP when measured on top of the polymer film, while this does not happen, as the initial value is comparable to that measured on glass. Finally yet importantly, the measurement reported below on a dye film excludes this too.

We conclude that optostimulation through the organic semiconductor layer leads to bigger changes than using the heating plate, both in terms of spectral shape ( $\Delta \mathrm{GP}$ variation) and decay kinetics, albeit the induced temperature rise is comparable: this suggests the presence of an additional excitation phenomenon.

Membrane Fluidity Assessment. As stated above, Laurdan is primarily sensitive to polarity, which in turn depends on the membrane order. To evaluate possible changes in the membrane order regardless of polarity, we probed directly the plasma membrane fluidity by measuring the fluorescence anisotropy of the membrane marker 1-(4trimethylamino)phenyl)-6-phenylhexa-1,3,5-triene (TMA$\mathrm{DPH}$ ) in living HEK-293 cells. TMA-DPH fluorescence lifetime is a good indicator of the microenvironment of the fluorophore, and earlier studies have shown that TMA-DPH exhibits different lifetimes in the liquid-ordered and liquiddisordered phases. ${ }^{64,65}$ As shown in Figure S8a, the decay of the fluorescence anisotropy in samples at room temperature and excited at $370 \mathrm{~nm}$ only exhibits a monoexponential decay ( $\tau \sim 1.5 \mathrm{~ns}$ ), characteristic of a gel (Lo) phase of the cell membrane. This was valid for both glass and P3HT:PCBM substrates. Heating of the samples with the Peltier plate or photoexcitation of the polymeric substrate using the CW laser leads to similar changes in the PL dynamics $(\tau \sim 1.1 \mathrm{~ns})$ indicating the formation of a more fluid phase in the plasma membrane following the temperature rise. Because the expected temperature rise in both situations is similar, the result is consistent with a thermally induced change of the membrane fluidity in both configurations, namely, the increase in membrane fluidity (lower order) with higher temperature.

\section{DISCUSSION}

Using two intramembrane fluorescent probes, we show that the cell temperature increase induces a structural relaxation of the plasma membrane toward a more fluid and less ordered phase, which favors water penetration. This offers an explanation for the observed temperature-induced capacitance enhancement, beyond the geometrical effect (smaller thickness and larger area). Water penetration in the membrane leads to higher dielectric screening, which is directly proportional to capacitance. Furthermore, we cannot exclude that water is also contributing to the reduction in electrical resistance across the membrane that was previously reported. ${ }^{16}$

Comparing the heating induced by a Peltier device with that induced by photoexcitation of a P3HT:PCBM layer, we find that photoexcitation is causing a larger (almost double) change in the observable linked to polarity of the cell membrane. As our direct membrane fluidity assessment with the probe TMA$\mathrm{DPH}$ is consistent with a thermally induced change of the membrane fluidity in both configurations (i.e., increase in membrane fluidity with higher temperature), we conjecture the reason behind the unexpected discrepancy between photoexcitation and heating to be because of the surface electrical charging. To evaluate this scenario, the P3HT:PCBM layer was replaced by a coloring pigment CI 15850 (D\&C Red No.7) film, a material that absorbs light in the same range of the organic semiconductor blend but does not support photogeneration of charges. We carefully controlled the deposition process of CI 15850 to obtain films with comparable optical density at the selected excitation wavelength $(\lambda=561 \mathrm{~nm}$, see Figure S1a), thus able to generate a temperature increase comparable to that of P3HT:PCBM. We verified this by measuring the bath temperature variation in the close proximity of the absorbing layer using the method of the calibrated pipette resistance ${ }^{66}$ (Figure S1b). As reported in Figure S9, we found that upon exciting the sample with the CW $561 \mathrm{~nm}$ laser, the green component in the Laurdan emission spectrum $(510 \mathrm{~nm})$ increases only to a modest extent. Deconvolution analysis yields the average relative area values reported in Table $S 5$, from which the calculated $\Delta \mathrm{GP}$ is found to be $-0.146(\Delta \mathrm{GP} / \mathrm{GP}=-0.9)$. This value is consistent with that obtained in the Peltier plate experiments $(\Delta \mathrm{GP}=-0.18 ; \Delta \mathrm{GP} / \mathrm{GP}=-1)$, where the only parameter in play is temperature, but it is significantly smaller than that measured for P3HT:PCBM $(\Delta \mathrm{GP}=-0.349 ; \Delta \mathrm{GP} / \mathrm{GP}=$ -1.8 ). The PL lifetime analysis (Figure S9c) leads to the same outcome. Kinetics fitted to double exponential decay curves display only slightly faster Laurdan lifetime when the $561 \mathrm{~nm}$ CW laser is switched on. In the measured values reported in Table 3, time constant $\tau_{2}$ drops by $2.4 \%$, while $\tau_{1}$ remains constant and $y_{0}$ decreases by $28 \%$. 
Table 3. Laurdan Decay Curve Average Parameters $(n=8)$ Obtained Exciting HEK-293 Cells Plated on CI 15850 Substrates $^{a}$

\begin{tabular}{|lll} 
& \multicolumn{1}{c}{ exc. $370 \mathrm{~nm}$} & exc. $370 \mathrm{~nm}+\mathrm{cw} 561 \mathrm{~nm}$ \\
$y_{0}$ & $0.14 \pm 0.003$ & $0.09 \pm 0.001$ \\
$\tau_{1}$ & $50 \pm 5$ ps $(\sim 53 \%)$ & $50 \pm 5$ ps $(\sim 57 \%)$ \\
$\tau_{2}$ & $438 \pm 9$ ps $(\sim 47 \%)$ & $427 \pm 8$ ps $(\sim 43 \%)$
\end{tabular}

${ }^{a}$ Kinetics were fitted to biexponential decay functions, with an offset $\left(y_{0}\right)$ accounting for the long lifetime component that falls outside our time-window.

We can thus conclude that photoexcitation of a "dielectric" film leads to smaller effect on the cell membrane compared to photoexcitation of a semiconductor film. A possible cause of such a discrepancy is the photogeneration of long-lived charges in the P3HT:PCBM film. To corroborate such a hypothesis, we first attempt to evaluate the charge photogeneration process in the P3HT:PCBM film interfaced with a liquid via an experimental protocol, which includes: (i) running a numerical simulation based on the drift-diffusion model; (ii) setting up a photoelectrochemical experiment to measure open-circuit PV; and (iii) measuring zeta surface potential.

The simulation reproduces the photoinduced charge carrier dynamics in a P3HT:PCBM film sandwiched between an ITO electrode and the electrolyte. The experimental observable is photovoltage as detected at the ITO electrode that accounts for the charge configuration at both interfaces. A key assumption in the model is the accumulation of photogenerated negative charges in proximity of the electrolyte because of oxygen trapping. ${ }^{34,67}$ The interface is far from an ideal flat plane, rather being a rough boundary defining a diffuse interface in the order of $10 \mathrm{~nm}$, in which hydrated oxygen and water clusters permeate the polymer free volume. The negative charges will be distributed within the diffuse interface at the electrolyte, while the positive charges spread in the polymer bulk. The simulation provides an estimation of the accumulated charge at the interface after $120 \mathrm{~s}$ of illumination of $\sigma=8 \times 10^{-2} \mathrm{Cm}^{-2}$, which is coherent with the one retrieved from the experimental PV (see Figure S10). The obtained value also matches well with the surface potential measurement, providing $V=-30 \mathrm{mV}$ at about $200 \mu \mathrm{m}$ from the surface of the sample, as visible in Figure S11. Once assessed the negative charging of the polymer surface, we discuss what this could do to our experiment on cells. The cell membrane adjacent to the polymer film surface rests on a protein layer that fills the cleft. At the close proximity of the interface, there will be a weak electric potential that we can estimate considering two disks with surface charge $\sigma$, of opposite sign, with an area equivalent to the cell one and separated by a distance $\delta x$. For distances $x$ from the surface such as $x \gg \delta x$, the electrostatic voltage is (see the Supporting Information)

$$
V(x)=-\frac{\sigma \cdot \delta x}{2 \varepsilon}\left(\frac{x}{\sqrt{R^{2}+x^{2}}}-1\right)
$$

where $\delta x$ is the effective separation of the negative and positive charge at the polymer electrolyte interface and $R=15 \mu \mathrm{m}$ is the cell radius. The distance $x$ corresponds to the cleft thickness for the adjacent side, and to the cell height for the opposite side, $x=0.01 \mu \mathrm{m}$ and $x=10 \mu \mathrm{m}$, respectively. If we assume $\delta x=1 \mathrm{~nm}$, we get $V_{\text {adj }} \approx-50 \mathrm{mV}$ and $V_{\text {opp }}=-30 \mathrm{mV}$. The corresponding electric field is in the order of $10^{3} \mathrm{~V} / \mathrm{m}$.
These numbers should not be considered as quantitative results, rather they suggest the existence of an electrical polarization that could act upon the CT emission from Laurdan, the water penetration in the membrane, the protein layer in the cleft, or the local $\mathrm{pH}^{68,69}$ At this stage, we cannot speculate further.

In conclusion, we showed that water penetration in the plasma membrane is likely the major contribution to the capacitance enhancement observed in cell's electrophysiology upon rising the temperature. Furthermore, we highlighted a peculiar effect associated with the photoexcitation of an organic semiconductor interface that may be reconciled to the steady-state charge generation following light absorption. We note here that reducing the light intensity does not allow to separate the photoinduced from the temperature-induced phenomena, as both have similar dependence. Albeit inspiring toward the understanding of the abiotic/biotic interface in general, this work explores the regime of long stimuli, in the tens of seconds, and high light intensity $\left(>1 \mathrm{~mW} / \mathrm{mm}^{2}\right)$ well separated from the short time stimulation and low intensity adopted in electrophysiology or possibly taking place in vivo. ${ }^{14}$

\section{ASSOCIATED CONTENT}

\section{Supporting Information}

The Supporting Information is available free of charge at https://pubs.acs.org/doi/10.1021/acs.jpcb.1c06054.

Supplementary discussion on finite element simulation of thermal diffusion (Figure S3) and drift-diffusion simulations; supplementary figures of UV-vis absorption and calibrated pipette measurements of the two employed photoactive layers (Figure S1); Laurdan decay fittings for different conditions and control measurements (Figures S2, S4-S7, and S9); TMA-DPH anisotropy measurements (Figure S8); PV measurements (Figure S10); zeta surface potential measurement (Figure S11); and Supplementary tables of Laurdan GP values (Tables S1-S5) (PDF)

\section{AUTHOR INFORMATION}

\section{Corresponding Authors}

Giuseppe Maria Paternò - Center for Nano Science and Technology@PoliMi, Istituto Italiano di Tecnologia, 20133 Milan, Italy; ○ orcid.org/0000-0003-2349-566X;

Email: giuseppe.paterno@iit.it

Guglielmo Lanzani - Department of Physics, Politecnico di Milano, 20133 Milan, Italy; Center for Nano Science and Technology@PoliMi, Istituto Italiano di Tecnologia, 20133 Milan, Italy; ○orcid.org/0000-0002-2442-4495;

Email: guglielmo.lanzani@iit.it

Cosimo D'Andrea - Department of Physics, Politecnico di Milano, 20133 Milan, Italy; Center for Nano Science and Technology@PoliMi, Istituto Italiano di Tecnologia, 20133 Milan, Italy; Email: cosimo.dandrea@polimi.it

\section{Authors}

Gaia Bondelli - Department of Physics, Politecnico di Milano, 20133 Milan, Italy; Center for Nano Science and Technology @PoliMi, Istituto Italiano di Tecnologia, 20133 Milan, Italy

Samim Sardar - Center for Nano Science and Technology@ PoliMi, Istituto Italiano di Tecnologia, 20133 Milan, Italy; (1) orcid.org/0000-0003-1783-6974 
Greta Chiaravalli - Department of Physics, Politecnico di Milano, 20133 Milan, Italy; Center for Nano Science and Technology@PoliMi, Istituto Italiano di Tecnologia, 20133 Milan, Italy

Vito Vurro - Center for Nano Science and Technology @ PoliMi, Istituto Italiano di Tecnologia, 20133 Milan, Italy

Complete contact information is available at:

https://pubs.acs.org/10.1021/acs.jpcb.1c06054

\section{Author Contributions}

S.S. optimized the TRPL setup. S.S. and G.B. performed TRPL experiments. G.B. fabricated the polymeric substrates and cell samples, performed experiments with a confocal microscope, analyzed the data, and wrote the manuscript, with input from all authors. G.C. performed the drift-diffusion simulations and the electrochemical and zeta surface potential measurements. V.V. performed the thermal increase simulation and conducted calibrated pipette measurements. G.L., G.M.P, and C.D.A. conceived and coordinated the project, planned experiments, and provided resources. All authors contributed to the interpretation and discussion of results.

Notes

The authors declare no competing financial interest.

\section{ACKNOWLEDGMENTS}

G.M.P. thanks Fondazione Cariplo (Grant No. 2018-0979) for financial support.

\section{REFERENCES}

(1) Potter, S. M. Distributed Processing in Cultured Neuronal Networks. Prog. Brain Res. 2001, 130, 49-62.

(2) Manfredi, G.; Lodola, F.; Paternó, G. M.; Vurro, V.; Baldelli, P.; Benfenati, F.; Lanzani, G. The Physics of Plasma Membrane Photostimulation. APL Mater. 2021, 9, No. 030901.

(3) DiFrancesco, M. L.; Lodola, F.; Colombo, E.; Maragliano, L.; Bramini, M.; Paternò, G. M.; Baldelli, P.; Serra, M. D.; Lunelli, L.; Marchioretto, M.; et al. Neuronal Firing Modulation by a MembraneTargeted Photoswitch. Nat. Nanotechnol. 2020, 15, 296-306.

(4) Paternò, G. M.; Colombo, E.; Vurro, V.; Lodola, F.; Cimò, S.; Sesti, V.; Molotokaite, E.; Bramini, M.; Ganzer, L.; Fazzi, D.; D’Andrea, C.; Benfenati, F.; Bertarelli, C.; Lanzani, G. Membrane Environment Enables Ultrafast Isomerization of Amphiphilic Azobenzene. Adv. Sci. 2020, 7, No. 1903241.

(5) Paternò, G. M.; Bondelli, G.; Sakai, V. G.; Sesti, V.; Bertarelli, C.; Lanzani, G. The Effect of an Intramembrane Light-Actuator on the Dynamics of Phospholipids in Model Membranes and Intact Cells. Langmuir 2020, 36, 11517-11527.

(6) Shapiro, M. G.; Homma, K.; Villarreal, S.; Richter, C. P.; Bezanilla, F. Infrared Light Excites Cells by Changing Their Electrical Capacitance. Nat. Commun. 2012, 3, 736.

(7) Bareket-Keren, L.; Hanein, Y. Novel Interfaces for Light Directed Neuronal Stimulation: Advances and Challenges. Int. J. Nanomed. 2014, 9, 65-83.

(8) Martino, N.; Ghezzi, D.; Benfenati, F.; Lanzani, G.; Antognazza, M. R. Organic Semiconductors for Artificial Vision. J. Mater. Chem. B 2013, 1, 3768-3780.

(9) Benfenati, V.; Martino, N.; Antognazza, M. R.; Pistone, A.; Toffanin, S.; Ferroni, S.; Lanzani, G.; Muccini, M. Photostimulation of Whole-Cell Conductance in Primary Rat Neocortical Astrocytes Mediated by Organic Semiconducting Thin Films. Adv. Healthcare Mater. 2014, 3, 392-399.

(10) Ghezzi, D.; Antognazza, M. R.; MacCarone, R.; Bellani, S.; Lanzarini, E.; Martino, N.; Mete, M.; Pertile, G.; Bisti, S.; Lanzani, G.; Benfenati, F. A Polymer Optoelectronic Interface Restores Light Sensitivity in Blind Rat Retinas. Nat. Photonics 2013, 7, 400-406.
(11) Ghezzi, D.; Antognazza, M. R.; Dal Maschio, M.; Lanzarini, E.; Benfenati, F.; Lanzani, G. A Hybrid Bioorganic Interface for Neuronal Photoactivation. Nat. Commun. 2011, 2, 166.

(12) Dennler, G.; Scharber, M. C.; Brabec, C. J. Polymer-Fullerene Bulk-Heterojunction Solar Cells. Adv. Mater. 2009, 21, 1323-1338.

(13) Lanzani, G. Materials for Bioelectronics: Organic Electronics Meets Biology. Nat. Mater. 2014, 13, 775-776.

(14) Pappas, T. C.; Wickramanyake, W. M. S.; Jan, E.; Motamedi, M.; Brodwick, M.; Kotov, N. A. Nanoscale Engineering of a Cellular Interface with Semiconductor Nanoparticle Films for Photoelectric Stimulation of Neurons. Nano Lett. 2007, 7, 513-519.

(15) Gautam, V.; Rand, D.; Hanein, Y.; Narayan, K. S. A Polymer Optoelectronic Interface Provides Visual Cues to a Blind Retina. Adv. Mater. 2014, 26, 1751-1756.

(16) Martino, N.; Feyen, P.; Porro, M.; Bossio, C.; Zucchetti, E.; Ghezzi, D.; Benfenati, F.; Lanzani, G.; Antognazza, M. R. Photothermal Cellular Stimulation in Functional Bio-Polymer Interfaces. Sci. Rep. 2015, 5, 8911.

(17) Feyen, P.; Colombo, E.; Endeman, D.; Nova, M.; Laudato, L.; Martino, N.; Antognazza, M. R.; Lanzani, G.; Benfenati, F.; Ghezzi, D. Light-Evoked Hyperpolarization and Silencing of Neurons by Conjugated Polymers. Sci. Rep. 2016, 6, 22718.

(18) Maya-Vetencourt, J. F.; Ghezzi, D.; Antognazza, M. R.; Colombo, E.; Mete, M.; Feyen, P.; Desii, A.; Buschiazzo, A.; Di Paolo, M.; Di Marco, S.; et al. A Fully Organic Retinal Prosthesis Restores Vision in a Rat Model of Degenerative Blindness. Nat. Mater. 2017, $16,681-689$.

(19) Rand, D.; Jakešová, M.; Lubin, G.; Vèbraite, I.; David-Pur, M.; Đerek, V.; Cramer, T.; Sariciftci, N. S.; Hanein, Y.; Głowacki, E. D. Direct Electrical Neurostimulation with Organic Pigment Photocapacitors. Adv. Mater. 2018, 30, No. 1707292.

(20) Jakešová, M.; Silverå Ejneby, M.; Đerek, V.; Schmidt, T.; Gryszel, M.; Brask, J.; Schindl, R.; Simon, D. T.; Berggren, M.; Elinder, F.; Głowacki, E. D. Optoelectronic Control of Single Cells Using Organic Photocapacitors. Sci. Adv. 2019, 5, No. eaav5265.

(21) Maya-Vetencourt, J. F.; Manfredi, G.; Mete, M.; Colombo, E.; Bramini, M.; Di Marco, S.; Shmal, D.; Mantero, G.; Dipalo, M.; Rocchi, A.; et al. Subretinally Injected Semiconducting Polymer Nanoparticles Rescue Vision in a Rat Model of Retinal Dystrophy. Nat. Nanotechnol. 2020, 15, 698-708.

(22) Tortiglione, C.; Antognazza, M. R.; Tino, A.; Bossio, C.; Marchesano, V.; Bauduin, A.; Zangoli, M.; Morata, S. V.; Lanzani, G. Semiconducting Polymers Are Light Nanotransducers in Eyeless Animals. Sci. Adv. 2017, 3, No. e1601699.

(23) Scanziani, M.; Häusser, M. Electrophysiology in the Age of Light. Nature 2009, 461, 930-939.

(24) Vurro, V.; Scaccabarozzi, A. D.; Lodola, F.; Storti, F.; Marangi, F.; Ross, A. M.; Paternò, G. M.; Scotognella, F.; Criante, L.; Caironi, M.; Lanzani, G. A Polymer Blend Substrate for Skeletal Muscle Cells Alignment and Photostimulation. Adv. Photonics Res. 2021, 2, No. 2000103.

(25) Vurro, V.; Bondelli, G.; Sesti, V.; Lodola, F.; Paternò, G. M.; Lanzani, G.; Bertarelli, C. Molecular Design of Amphiphilic Plasma Membrane-Targeted Azobenzenes for Nongenetic Optical Stimulation. Front. Mater. 2021, 7, 472.

(26) Carvalho-de-Souza, J. L.; Treger, J. S.; Dang, B.; Kent, S. B. H.; Pepperberg, D. R.; Bezanilla, F. Photosensitivity of Neurons Enabled by Cell-Targeted Gold Nanoparticles. Neuron 2015, 86, 207-217.

(27) Plaksin, M.; Kimmel, E.; Shoham, S. Correspondence: Revisiting the Theoretical Cell Membrane Thermal Capacitance Response. Nat. Commun. 2017, 8, 1431.

(28) Plaksin, M.; Shapira, E.; Kimmel, E.; Shoham, S. Thermal Transients Excite Neurons through Universal Intramembrane Mechanoelectrical Effects. Phys. Rev. X 2018, 8, 11043.

(29) Heimburg, T. Thermal Biophysics of Membranes; Wiley-VCH Verlag GmbH \& Co. KGaA:Weinheim, Germany, 2008.

(30) Heimburg, T. The Capacitance and Electromechanical Coupling of Lipid Membranes Close to Transitions: The Effect of Electrostriction. Biophys. J. 2012, 103, 918-929. 
(31) Owen, D. M.; Rentero, C.; Magenau, A.; Abu-Siniyeh, A.; Gaus, K. Quantitative Imaging of Membrane Lipid Order in Cells and Organisms. Nat. Protoc. 2012, 7, 24-35.

(32) Prendergast, F. G.; Haugland, R. P.; Callahan, P. J. 1-[4(Trimethylamino)Phenyl]-6-Phenylhexa-1,3,5-Triene: Synthesis, Fluorescence Properties, and Use as a Fluorescence Probe of Lipid Bilayers. Biochemistry 1981, 20, 7333-7338.

(33) Illinger, D.; Kuhry, J. G. The Kinetic Aspects of Intracellular Fluorescence Labeling with TMA-DPH Support the Maturation Model for Endocytosis in L929 Cells. J. Cell Biol. 1994, 125, 783794.

(34) Bellani, S.; Fazzi, D.; Bruno, P.; Giussani, E.; Canesi, E. V.; Lanzani, G.; Antognazza, M. R. Reversible P3HT/Oxygen Charge Transfer Complex Identification in Thin Films Exposed to Direct Contact with Water. J. Phys. Chem. C 2014, 118, 6291-6299.

(35) Singer, S. J.; Nicolson, G. L. The Fluid Mosaic Model of the Structure of Cell Membranes. Science 1972, 175, 720-731.

(36) Goñi, F. M. The Basic Structure and Dynamics of Cell Membranes: An Update of the Singer-Nicolson Model. Biochim. Biophys. Acta, Biomembr. 2014, 1838, 1467-1476.

(37) Sezgin, E.; Levental, I.; Mayor, S.; Eggeling, C. The Mystery of Membrane Organization: Composition, Regulation and Roles of Lipid Rafts. Nat. Rev. Mol. Cell Biol. 2017, 18, 361-374.

(38) Suhaj, A.; Gowland, D.; Bonini, N.; Owen, D. M.; Lorenz, C. D. Laurdan and Di-4-ANEPPDHQ Influence the Properties of Lipid Membranes: A Classical Molecular Dynamics and Fluorescence Study. J. Phys. Chem. B 2020, 124, 11419-11430.

(39) Simons, K.; Ikonen, E. Functional Rafts in Cell Membranes. Nature 1997, 387, 569-572.

(40) Heimburg, T. Physical Properties of Biological Membranes. 2009, arXiv:0902.2454.

(41) van Meer, G.; Voelker, D. R.; Feigenson, G. W. Membrane Lipids: Where They Are and How They Behave. Nat. Rev. Mol. Cell Biol. 2008, 9, 112-124.

(42) Goshima, G.; Kiyomitsu, T.; Yoda, K.; Yanagida, M. Human Centromere Chromatin Protein HMis12, Essential for Equal Segregation, Is Independent of CENP-A Loading Pathway. J. Cell Biol. 2003, 160, 25-39.

(43) Weber, G.; Farris, F. J. Synthesis and Spectral Properties of a Hydrophobic Fluorescent Probe: 6-Propionyl-2-(Dimethylamino)Naphthalene. Biochemistry 1979, 18, 3075-3078.

(44) Macgregor, R. B.; Weber, G. Fluorophores in Polar Media: Spectral Effects of the Langevin Distribution of Electrostatic Interactions. Ann. N. Y. Acad. Sci. 1981, 366, 140-154.

(45) Parasassi, T.; Krasnowska, E. K.; Bagatolli, L.; Gratton, E. Laurdan and Prodan as Polarity-Sensitive Fluorescent Membrane Probes. J. Fluoresc. 1998, 8, 365-373.

(46) Viard, M.; Gallay, J.; Vincent, M.; Meyer, O.; Robert, B.; Paternostre, M. Laurdan Solvatochromism: Solvent Dielectric Relaxation and Intramolecular Excited-State Reaction. Biophys. J. 1997, 73, 2221-2234.

(47) Vincent, M.; de Foresta, B.; Gallay, J. Nanosecond Dynamics of a Mimicked Membrane-Water Interface Observed by Time-Resolved Stokes Shift of LAURDAN. Biophys. J. 2005, 88, 4337-4350.

(48) Parasassi, T.; De Stasio, G.; Ravagnan, G.; Rusch, R. M.; Gratton, E. Quantitation of Lipid Phases in Phospholipid Vesicles by the Generalized Polarization of Laurdan Fluorescence. Biophys. J. 1991, 60, 179-189.

(49) Klymchenko, A. S. Solvatochromic and Fluorogenic Dyes as Environment-Sensitive Probes: Design and Biological Applications. Acc. Chem. Res. 2017, 50, 366-375.

(50) Parasassi, T.; Gratton, E. Membrane Lipid Domains and Dynamics as Detected by Laurdan Fluorescence. J. Fluoresc. 1995, 5, $59-69$.

(51) Krasnowska, E. K.; Gratton, E.; Parasassi, T. Prodan as a Membrane Surface Fluorescence Probe: Partitioning between Water and Phospholipid Phases. Biophys. J. 1998, 74, 1984-1993.

(52) Bagatolli, L. A.; Parasassi, T.; Fidelio, G. D.; Gratton, E. A Model for the Interaction of 6-Lauroyl-2-(N,N-Dimethylamino)-
Naphthalene with Lipid Environments: Implications for Spectral Properties. Photochem. Photobiol. 1999, 70, 557.

(53) Parasassi, T.; Gratton, E.; Yu, W. M.; Wilson, P.; Levi, M. TwoPhoton Fluorescence Microscopy of Laurdan Generalized Polarization Domains in Model and Natural Membranes. Biophys. J. 1997, 72, 2413-2429.

(54) Parasassi, T.; De Stasio, G.; d'Ubaldo, A.; Gratton, E. Phase Fluctuation in Phospholipid Membranes Revealed by Laurdan Fluorescence. Biophys. J. 1990, 57, 1179-1186.

(55) Mély, Y.; Duportail, G.; Bagatolli, L. A. Fluorescent Methods to Study Biological Membranes; Springer Berlin Heidelberg: Berlin, 2013.

(56) Gaus, K.; Zech, T.; Harder, T. Visualizing Membrane Microdomains by Laurdan 2-Photon Microscopy. Mol. Membr. Biol. 2006, 23, 41-48.

(57) Golfetto, O.; Hinde, E.; Gratton, E. Laurdan Fluorescence Lifetime Discriminates Cholesterol Content from Changes in Fluidity in Living Cell Membranes. Biophys. J. 2013, 104, 1238-1247.

(58) Vequi-Suplicy, C. C.; Lamy, M. T.; Marquezin, C. A. The New Fluorescent Membrane Probe Ahba: A Comparative Study with the Largely Used Laurdan. J. Fluoresc. 2013, 23, 479-486.

(59) Ma, Y.; Benda, A.; Kwiatek, J.; Owen, D. M.; Gaus, K. TimeResolved Laurdan Fluorescence Reveals Insights into Membrane Viscosity and Hydration Levels. Biophys. J. 2018, 115, 1498-1508.

(60) Noutsi, P.; Gratton, E.; Chaieb, S. Assessment of Membrane Fluidity Fluctuations during Cellular Development Reveals Time and Cell Type Specificity. PLoS One 2016, 11, No. e0158313.

(61) Jurkiewicz, P.; Sýkora, J.; Olzyńska, A.; Humpolíčková, J.; Hof, M. Solvent Relaxation in Phospholipid Bilayers: Principles and Recent Applications. J. Fluoresc. 2005, 15, 883-894.

(62) Sýkora, J.; Kapusta, P.; Fidler, V.; Hof, M. On What Time Scale Does Solvent Relaxation in Phospholipid Bilayers Happen? Langmuir 2002, 18, 571-574.

(63) McNeill, C. R.; Abrusci, A.; Hwang, I.; Ruderer, M. A.; MüllerBuschbaum, P.; Greenham, N. C. Photophysics and Photocurrent Generation in Polythiophene/Polyfluorene Copolymer Blends. Adv. Funct. Mater. 2009, 19, 3103-3111.

(64) Barrow, D. A.; Lentz, B. R. Membrane structural domains. Resolution limits using diphenylhexatriene fluorescence decay. Biophys. J. 1985, 48, 221-234.

(65) Sinha, M.; Mishra, S.; Joshi, P. G. Liquid-Ordered Microdomains in Lipid Rafts and Plasma Membrane of U-87 MG Cells: A Time-Resolved Fluorescence Study. Eur. Biophys. J. 2003, 32, 381391.

(66) Yao, J.; Liu, B.; Qin, F. Rapid Temperature Jump by Infrared Diode Laser Irradiation for Patch-Clamp Studies. Biophys. J. 2009, 96, 3611-3619.

(67) Mosconi, E.; Salvatori, P.; Saba, M. I.; Mattoni, A.; Bellani, S.; Bruni, F.; Santiago Gonzalez, B.; Antognazza, M. R.; Brovelli, S.; Lanzani, G.; et al. Surface Polarization Drives Photoinduced Charge Separation at the P3HT/Water Interface. ACS Energy Lett. 2016, 1, 454-463.

(68) Wan, A. M. D.; Schur, R. M.; Ober, C. K.; Fischbach, C.; Gourdon, D.; Malliaras, G. G. Electrical Control of Protein Conformation. Adv. Mater. 2012, 24, 2501-2505.

(69) Lodola, F.; Martino, N.; Tullii, G.; Lanzani, G.; Antognazza, M. R. Conjugated Polymers Mediate Effective Activation of the Mammalian Ion Channel Transient Receptor Potential Vanilloid 1. Sci. Rep. 2017, 7, 8477. 